To be submitted to Expert Review of Molecular Diagnostics

\title{
Blood Biomarkers and Treatment response in Major Depression
}

Authors:

Cristina Mora ${ }^{1}$, Valentina Zonca ${ }^{1,2}$, Marco A. Riva ${ }^{2}$ and Annamaria Cattaneo ${ }^{1,3}$

${ }^{1}$ Biological Psychiatry Unit, IRCCS Fatebenefratelli S. Giovanni di Dio, Brescia;

${ }^{2}$ University of Milan, Department of Pharmacological and Biomolecular Sciences, Milan, Italy;

${ }^{3}$ Stress, Psychiatry and Immunology Laboratory, Department of Psychological Medicine, Institute of Psychiatry, King's College, London.

\section{Corresponding Author}

Prof. Marco A Riva

Department of Pharmacological and Biomolecular Sciences,

University of Milan, Milan, Italy;

Email: m.riva@unimi.it 


\begin{abstract}
Introduction: Millions of people worldwide suffer from depression, but despite advances in pharmacological therapies, many patients do not experience symptomatic remission or treatment response, even after treatments with several medications. As such, there is an urgent need to identify biomarkers that can not only predict the treatment response, but also allow a rational selection of optimal therapy for each patient leading to a precision medicine.
\end{abstract}

Areas covered: This review examines the recent findings, coming from different "omic sciences", in human blood-based biomarkers associated with antidepressant treatment response with particular attention on genetic/epigenetic and biochemical biomarkers. Specific emphasis will be placed on key molecules related to neuroplasticity and inflammation because of their involvement in the pathophysiology of depression and antidepressant treatment response.

Expert commentary: Biomarkers identification is still an ongoing work. Indeed, to date, no biomarkers have sufficiently proven specificity, sensitivity and reproducibility to be used in the clinical setting. However, "omic" approaches hold great promise in identifying multiple features for predicting antidepressant response, making possible a personalized treatment strategy for each patient, and thereby assist with quick and efficacious responsiveness. But for this to be possible, it is necessary that future studies take an integrative approach that include clinical assessment, environment influences and molecular and biological biomarkers.

KEYWORDS: Antidepressants, Biomarkers, Cytochrome, Cytokines, Epigenomics, Gene expression profile, Major depressive disorder, Metabolomics, Neurotrophic Factors, Pharmacogenomics, Proteomics. 


\section{BACKGROUND}

According to the latest available estimates of the prevalence of depression (2015), globally, 322 million people suffer from this disorder (equivalent to $4.4 \%$ of the world's population), with a higher prevalence among females than males (5.1\% and 3.6\% respectively) (Depression and Other Common Mental Disorders: Global Health Estimates. Geneva: World Health Organization; 2017. Licence: CC BY-NC-SA 3.0 IGO). Moreover, from a global perspective, depressive disorder is predicted to be the leading cause of disease burden worldwide, measured in disability adjusted life years, by the year 2030 (WHO, The global burden of disease: 2004 update., 2004, World Health Organization).

Depressive disorders include a heterogeneous spectrum of clinical conditions which are characterized by several common features such as the presence of sad, empty, or irritable mood, accompanied by somatic and cognitive changes that significantly affect the individual's capacity to function [1]. Especially when depressive episodes are long-lasting and with moderate or severe intensity, the disorder becomes a serious health condition, that can possibly lead to suicide. Indeed, according to an estimation by the World Health Organization that about 788.000 people died due to suicide, denoting the need of urgent actions (Depression and Other Common Mental Disorders: Global Health Estimates. Geneva: World Health Organization; 2017. Licence: CC BY-NC-SA 3.0 IGO).

Meta-analysis of family-related studies indicated an estimated odds ratio for increased risk for MDD in firstdegree relatives of MDD probands of 2.84, and an estimated heritability for MDD of about the 30\% [2]. However, there is a general consensus about the great complexity of the aetiopathogenesis, where it is now clear that MDD is the result of a combined contribution of several vulnerability genes, of the environment and in particular of adverse events and also of Gene-Environment interactions (G X E). Indeed, adverse life events, especially early in life, have been consistently shown to strongly increase not only the risk for mood and anxiety disorders in large epidemiological studies but also influence the treatment outcome $[3,4,5,6,7]$.

Although different pharmacological therapies are available, there is still significant variability in the response to antidepressant treatment response: almost $60 \%$ of patients do not recover following a single antidepressant trial, and $20 \%$ of these patients fail to respond to any interventions [8]. Indeed, the most effective antidepressant medication for each patient can presently only be identified through a trial and error strategy [9]: this imply the lengthening of remission, patient's suffering, poor compliance, appearance of treatment adverse effects, worsening of suicidal ideation and high costs for society [10,11].

According to this, there is an urgent need to identify biomarkers that may be able to predict the future treatment response, and ideally biomarkers that can detect the best antidepressant drug for each individual patient, which will eventually lead to a personalized medicine. Among all the possible biomarkers, peripheral blood biomarkers represent the most accessible ones, given their suitability and ease of collection in clinical practice. The discovery of such biomarkers will help: i) the development of a precision medicine in psychiatry; ii) the improvement of the effectiveness of treatment and ultimately the remission and iii) the discovery of new targets for the development of novel antidepressant drugs. 
Based on this background information, here we will review the literature on blood-based biomarkers that have been, up to date, associated with treatment response in depressed patients.

In particular, we will primarily focus on genetic/epigenetic (polymorphisms, micro-RNA, mRNA, DNA methylation, histones) and biochemical biomarkers, mentioning also more recent findings from proteomic and metabolomic approaches. Particular emphasis-will be placed on two candidate biological systems, namely the immune/inflammatory and neuroendocrine systems, because of large amount of data supporting their involvement in the pathophysiology of depression and, most importantly, in treatment response $[12,13,14,15]$. This review focuses on recent findings (over the past 15 years approximately). We used Pubmed database to conduct a careful literature review, with the following key-words combinations: "depression","antidepressant response, "blood”, "biomarkers”,"gene expression,“mRNA, "miRNA, epigenetic,"DNA methylation,“histone modification, "cytokines", "cytochrome", "pharmacogenetic", "inflammation", "metabolomic" and "proteomic". We used PubMed automatic filters to select human studies in the English language published since 2000. We then selected most relevant articles/review with a manual screening of the abstracts.

\subsection{Available antidepressant strategies and current biomarkers of treatment response}

The Guidelines for the management of patients with MDD indicate the use of second-generation antidepressant drugs as first-line treatments for patients with an episode of moderate or greater severity (as determined by symptom scales and/or functional impairment) [16]. The selective serotonin reuptake inhibitors (SSRIs), serotonin noradrenaline reuptake inhibitors (SNRIs), and other antidepressant drugs including agomelatine, bupropion, mirtazapine and vortioxetine remain first-line recommendations for pharmacotherapy for MDD. Recommended second-line agents include TCAs, trazodone, levomilnacipran and vilazodone, despite their side effects. Third-line recommendations include MAO inhibitors and reboxetine. Furthermore, in order to achieve clinical improvement, particularly in the context of treatment-resistant depression, the NMDA antagonist Ketamine, used at sub-anaesthetic dose, has emerged as a potential new option, which may produce a rapid antidepressant response [17].

Many clinical factors and dimensions (including cognitive dysfunction, sleep disturbance, and somatic symptoms) as well as medication features (efficacy, tolerability, drug-drug interactions) can influence the choice of a first-line antidepressants. Thus, the selection of a specific antidepressant drug should involve a personalized assessment for each patient.

However, despite this high number of antidepressant drugs available, two thirds of MDD patients fail to achieve remission after an initial treatment, and almost one third fail to achieve remission even after four consecutive treatment trials [18].

This is in part associated to the fact that the diagnostic criteria for MDD is based on symptomatology assessment that may be present in other conditions thus leading to potential misdiagnoses: indeed, in only about $40-50 \%$ of patients the first diagnosis is then confirmed by further assessments [19], and, this may lead to inadequate pharmacological treatments. 
Furthermore, we need to consider that the individual variability to antidepressant response is also the consequence of an interplay between clinical factors and biological pathways, as well as with the environment [20]. For example, it has been clearly demonstrated that patients with a history of childhood trauma and other adversities, especially early in life, are also those with a low probability to respond to the first trials of antidepressant drugs or not respond at all to any kind of antidepressant therapy [5,6,7]. As such, there is an increasing interest in the identification of objective and reproducible criteria for the correct diagnosis of depression. Traditional assessment tools are based on clinician- or patient self-administered interview that have reasonable clinical accuracy depending on assessments scores, but they may lead to a wrong diagnosis, which may lead to wrong pharmacological treatment also due to the presence of overlapping symptoms across diagnosis [1].

Laboratory methods have been also settled to better improve the diagnosis procedure of depression and of its endophenotypes. They are based on the assumption that a number of biological pathways are altered in depressed patients (such as the inflammatory, neurotrophic, metabolic and hypothalamic-pituitary-adrenal HPA axis), thus diagnostic tests could be represented by the measurement of biomarkers reflecting the key biological pathways altered in depression, for example, the dexamethasone suppression test (DST), serum levels of BDNF and blood levels of cytokines. However, each test individually could explain a very modest proportion of the variance in depression risk.

New laboratory methods based on genomics, transcriptomics, proteomics and metabolomics may allow analysis hypothesis-free to identify new biomarker panels also for biologically defined MDD subtypes.

To date, we are aware of the presence of one test for depression that however is not yet used in the clinical setting: it is a multi-assay serum-based test that measures a panel of nine serum markers, derived from an earlier evaluation of 100 markers. Biomarker data were used to develop a logistic regression model that included gender and BMI in a manner that allowed for their interaction with the biochemical analytes (MDDScore) that distinguished MDD patients versus healthy controls [21]. However, in its present form, the test has some limitations including the inability to dissect unipolar versus bipolar depression [22]. The development of tests able to improve a correct diagnosis from the beginning will also improve the clinical outcome as pharmacological treatments will be better administered from the beginning.

The identification of biomarkers for treatment response is another a prerequisite for improving the efficacy of pharmacological treatment in patients with MDD and it will require identification of biomarkers of treatment response. A biomarker is defined as a characteristic that can be objectively measured and evaluated as an indicator of normal biologic processes, pathogenic processes, or pharmacologic responses to a therapeutic intervention [23].

Unfortunately, since we cannot yet predict the likelihood of response of an individual patient to a specific drug, there is an urgent need to identify biomarkers that, when assessed at baseline, may change future response to treatment (predictors), as well as biomarkers that are targeted by antidepressant, and change longitudinally during antidepressant treatment (mediators) $[18,20,24,25,26$,$] . Among these, predictors of response are more$ clinically relevant as they can help in improving patient's care. 
The first challenge for the identification of biomarkers associated with treatment response is the selection of the tissue/specimen in which the biomarkers can be measured. Biological fluids are the most practical tissues for patient sampling, particularly peripheral blood samples, as they are easy and quick to retrieve, although some limitations exist [27]. Moreover, blood samples allow simultaneously investigation of several different biomarkers, provide high concentrations and quality of DNA and RNA, and cellular components of peripheral blood (such as lymphocytes, monocytes and leukocytes) are suitable for functional studies [20].

In this article, we will provide an overview on the most promising biomarkers for antidepressant treatment response that could be used in the clinical practice. We provide evidence from different biological parameters, including polymorphisms, gene expression (mRNA levels), epigenetics, protein levels and metabolomic markers (Figure 1). 
Figure 1. A global approach to discover biomarkers for predicting variation in a subject's response to treatment.

\section{HOW GENETIC VARIANTS WITHIN CYTOCHROME GENES CAN INFLUENCE THE ANTIDEPRESSANT TREATMENT RESPONSE}

It is well known that drug blood concentration depends on intrinsic feature of the compound as well as the functional activity of the enzymes involved in its metabolism. These enzymes belong to the cytochrome P450 (CYP) superfamily, the major class implicated in drugs metabolism, accounting for about 50 different isoforms. Enzymes belonging to the CYP450 family oxidize and reduce several molecules, turning them into inactive or active compound. In this context, recent studies have tried to use the CYP variants to establish a personalized antidepressant prescription, and correspondent optimal dose. It is important to remember that the successful outcome of a therapy is not only influenced by CYP genetic variants, but also by others factors, such as food and concomitant drug administration, which have the potential to affect CYP enzymatic activity. It is noteworthy that antidepressant drugs are metabolised by different cytochrome isoforms and thus, the existence of SNPs can of course influence the correct pharmacological action only of the drug which is metabolised by that specific cytochrome isoforms; for this reason, the knowledge of cytochromes and SNPs is a key issue for personalization of therapy.

The main cytochromes involved into the metabolic pathways of antidepressant drugs are the isoforms CYP2D6 and CYP2C19. The importance of these isoforms has been recently demonstrated as they have been implied in clinical applications [28], moreover CYP2D6 and CYP2C19 genotyping test has been recently approved by the Food Drug Administration (FDA). Beyond CYP2D6 and CYP2C19, other CYP isoforms, namely CYP2C9, CYP2B6 and CYP1A2 are relevant in the metabolism of antidepressants, although they affect only few antidepressant drugs.

The antidepressant drugs currently used in the clinical setting and the CYPs isoforms that are involved in their metabolism are reported in table 1 .

The introduction of pharmacogenomic tests (such as amplichiptm cyp450 and infiniti cyp450), aiming at identify cytochromes' polymorphisms, has been an important step towards personalized medicine. The underlying rationale is the identification of the patient-specific metabolic profile, in order to design the best therapeutic approach according to the metabolic rate of a specific compounds. The desired end point would be the reduction of side effects and the adjustment of plasma drug concentration. The studies in this area have shown differences in the side-effect profile and drug concentration. However, during the last years, a few clinical trials have taken into account the general treatment efficacy, by assessing patients' improvements comparing different time points of the hdrs-17 scores between tau (treatment-as-usual) patients and those who were treated with a pharmacogenomic-guided treatment (as reviewed in the following articles: [29,30,31]. The results of guided treatments show promising results as well as the tested patients show better clinical outcomes 
compared to tau subjects. Although guided prescription might help improve both efficacy and tolerability, the available clinical trials have some limitations and should be replicated in a more comprehensive way, in order to understand different source of variabilities such age, sex and ethnicity.

The advantages of this pharmacogenomic analysis are the personalization of psychiatric treatments which can identify in advance the more effective medications and the needed dose adjustments, or it might drastically reduce the meaningful adverse reactions. On the other hand, the drawbacks principally lie in the higher costs of such pharmacogenomic tools, even though they have been reduced in the last years and are hopefully going to be downsized even within the years to come. Moreover, some clinicians are still reluctant to follow the pharmacogenomic test results, still prescribing medications with a high rate of contraindications for the patients (an example can be seen in the work [31]).

\section{Table 1. Current antidepressant drug used in therapy.}

The following paragraphs illustrates the implications of CYP2D6, CYP2C19, CYP2A1 and ABCB1 polymorphism on drugs bioavailability and on clinical antidepressant outcome.

\subsection{CYP2D6}

CYP2D6 accounts for the 25\% of all the drugs metabolized by the CYP family [32] and it is mainly involved in IMAOs' and TCAs' metabolism. The most common allele variant in Caucasian people is the CYP2D6*4 (allele frequency 20\%) and it has been associated with a poor metabolizer phenotype [33]. This means that the antidepressant blood concentration of individuals carrying this variant is overall higher than subjects carrying the wild-type variant (CYP2D6*1).

Another variant widely investigated in relation to paroxetine and venlafaxine treatment is CYP2D6*10B. It has been shown that in an Asian cohort of patients the high blood levels of paroxetine were associated to the $* 10 \mathrm{~B}$ variant $[34,35]$. It is important to notice that this variant does not affect drug concentration at the steady state, although the amount of paroxetine in blood after a single dose is increased in subjects with the *10B variant $[34,36]$. Moreover, the blood concentration had a more significant increment at higher dosages and this was linked to the inhibitory effect of paroxetine on CYP2D6 enzymatic activity. These findings are supported by other studies showing increased levels of venlafaxine in the blood of subjects carrying one of two copies of the $* 10$ allele [37].

It has been also reported that pregnancy, and in particular hormones during pregnancy, can influence the metabolic activity of the cytochrome CYP2D6: indeed, several studies showed lower concentration levels of antidepressant drugs in the blood of pregnant women, suggesting that pregnancy related hormones might have a possible direct role in enhancing CYP2D6 metabolic enzymatic activity [38]. This effect has to be taken into consideration for the proper prescription of antidepressant drugs during pregnancy: clinicians should firstly 
consider to administrate antidepressant compounds that are not metabolised by CYP2D6 in high risk expectant mothers, and eventually adjust the drug dose.

On the clinical side, it is not clearly established the potential beneficial effects displayed by carriers of the different CYP2D6 polymorphisms in term of treatment response. The only study conducted to evaluate possible effect of CYP2D6 polymorphisms in influencing treatment response was a 8-weeks study which failed to identify any differences in the reduction of Hamilton Scale of Depression (HDRS) between CYP2D6 poor/intermediate metabolizer and CYP2D6 extensive/ultra-rapid metabolizer after administration of escitalopram and venlafaxine [39], suggesting that CYP2D6 variants cannot influence the antidepressant efficacy. Regarding side effects, in the escitalopram group, UMs and EMs had increased autonomic side effects (for example, sweating and gastrointestinal pain) than PMs and IMs; no differences were reported in the venlafaxine group [39].

\subsection{CYP2C19}

Cytochrome P2C19 is also involved in the antidepressant drugs metabolism after CYP2D6 and it is involved in the metabolism of several antidepressant agents, including sertraline, citalopram, escitalopram and venlafaxine. The wild-type variant is the allele $* 1$, whereas the most common recessive ones are the $* 2$ and *17 variants. In Europeans and Asians, the CYP2C19*2 variant is responsible for the poor-metabolizer phenotype [40] and it has assumed a significant clinical importance due to the fact that it may be associated with a greater reduction of depressive symptoms when compared to extensive-metabolizer related variant. The association between CYP2C19*2 and depressive symptoms is not clear, but it has been suggested that this variant can also be involved directly also in the regulation of the biotransformation of 5-HT [41].

CYP2C19*17 variant is correlated with a rapid metabolism of antidepressants as demonstrated by its increased activity both in vitro and in vivo. As a consequence, the reduced blood concentration of antidepressants metabolized by CYP2C19*17 can probably be associated with the lack of efficacy of antidepressant treatment [42]. Some studies investigated the differences among the allele *1 and the allele *17: De Vos and colleges shown that the $* 17$ variant was associated with a reduction in the metabolic ratio for amitriptyline and citalopram compared to $* 1$ genotype; as a consequence, they suggested an association between the CYP2C1917 allele and the sub-therapeutic levels of those drugs metabolised by CYP2C1917, like citalopram and amitriptyline [43]. However, the study focused on sertraline metabolism in relation to CYP2C19, failed to identify any significant changes [44]. Furthermore, the same 8 weeks' study previously described for CYP2D6, found contrasting differences between CYP2C19 poor/intermediate and CYP2C19 extensive/ultra-rapid metabolizer after administration of escitalopram and venlafaxine [39].

\subsection{CYP2A1}

Although no relevant allelic variants within the CYP2A1 have been discovered, CYP2A1 has been investigated for several years because of inter-individual variability in its activity which is mainly due to environmental factors, such as smoke $[28,45]$. More in details, it was showed that smoke increased CYP2A1 enzymatic 
activity. A study on the antidepressant trazodone showed increased stready-state blood levels of antidepressants in depressed non-smokers subjects, and the differences from smoker patients did not depend on the genetic variant [46]. In another study, the genetic variant *1F was associated with increased CYP1A2 activity in smokers [47]. On these bases, smoke might induce cytochrome 2A1 enzymatic activity thus resulting in lower drug plasma levels, higher clearance and decreased pharmacological activity of antidepressants metabolized by CYP1A2, including imipramine, amitriptyline, clomipramine, duloxetine, fluvoxamine and mirtazapine [45].

Taking into account the effect of smoking on CYP2A1 and considering the high rates of smokers in patients with psychiatric illnesses, it seems important to consider the possibility of monitoring cigarette consumption in depressed patients treated with drugs metabolized by CYP2A1, in order to prevent a possible lack of efficacy and the subsequent worsening of the depressive symptoms in these patients.

\subsection{ABCB1}

The pharmacological effect of the antidepressant drugs does not depend exclusively on blood concentration at the steady state, but also on brain concentration which is influenced by their ability to permeate the bloodbrain barrier (BBB). The permeability through the BBB is primarily regulated by the efflux pump Pglycoprotein (P-gp), which is actively involved in transporting the substrates against a concentration gradient out of the brain cell into the blood and increase the drug clearance from blood. The G-gp is a $170 \mathrm{kDa}$ glycoprotein encoded on chromosome 7 by the ABCB1 gene, also known as multidrug resistance 1 gene (MDR1); different pharmacogenomics studies have investigated the ABCB1 SNPs in order to correlate genetic variants, possibly associated with drug resistance, with clinical outcome. In case the antidepressant drug is also a substrate of the glycoprotein, ABCB1 SNPs can be relevant in the clinical practice if the antidepressant drug is a substrate of the glycoprotein. Indeed, studies conducted on ABCB1 gene in knock-out mice (i.e., abcb1b knock out mice) have discovered which drugs undergo the G-pg activity and Table 2 shows the most relevant substrates used in therapy.

The study conducted by Uhr and collaborators is one of the first study associating several SNPs within ABCB1 gene with different clinical outcomes after antidepressant treatment. They demonstrated the influence of the SNP rs2232583 on response to 4-weeks treatment with a G-pg substrate: subjects carrying the C genotype had a higher probability to be remitters as compared to those with the TT genotype [48]. On the other hand, no influence of the same SNP on treatment response has been detected in patients treated with mirtazapine, which is not subjected to the action of the glycoprotein [48]. Similar results were also reported by Sarginson and coworkers in a study on geriatric depression [49]. The authors focused their attention on several SNPs within the genes, finding that rs 10245483 contributed to the prediction of the clinical outcome to three different antidepressants: homozygotes of common allele responded significantly better to escitalopram and sertraline, whereas minor allele homozygotes, benefitted from venlafaxine treatment, that is not a G-pg substrate [50]. 
Table 2. Antidepressant drug divided into substrates and not substrates of the efflux pump Pglycoprotein

\section{EPIGENOME AS INTERFACE BETWEEN ENVIRONMENT AND GENOME: THE SIGNATURE THAT MAKE US UNIQUE ALSO IN TERM OF PERSONALIZED THERAPY}

Epigenetics refers to heritable phenotypic traits which occur without any alterations in DNA sequence and includes DNA methylation, post translational modification of histones and microRNAs [51].

Epigenetic mechanisms, in the context of antidepressant treatment response, are involved in two different aspects: the first one is the interplay between the environmental stimuli and our genome, mediated by epigenetic mechanisms; importantly, modifications induced by environment and involving epigenetic processes can induce long-term alterations of the expression of the methylated/un-methylated gene. The second one is the direct or indirect effect of the drugs in current therapeutic practice on epigenomic status, that in turn can influence the treatment response: as an example, several antidepressant drugs and mood stabilizers (i.e., valproate, imipramine, amitriptyline, clomipramine, fluoxetine, escitalopram, phenelzine, and tranylcypromine) were shown to exert part of their therapeutic action by targeting DNA methyltransferase I (DNMT1), histone deacetylase or altering methylation levels, which in turn can affect gene expression $[52,53,54]$. Hence, targeting selective epigenetic mechanisms with DNMT modulators or HDAC inhibitors is being considered as a possible new avenue for the development of novel antidepressant drugs [54,55,56,57]. On these bases, we will next discuss if and how epigenetic processes, and in particular DNA methylation, histone modifications and microRNAs (miRNAs), can be used as biomarkers for treatment response.

\subsection{DNA methylation}

DNA Methylation, which represents the most investigated epigenetic mechanism, refers to the addition of methyl groups in the $5^{\text {th }}$ position of a Cytosine to form 5-methylcytosine $(5 \mathrm{mC})$, a reaction that is catalyzed by members of the DNA methyltransferase (DNMT) family [58]. From a clinical perspective, biomarkers associated with DNA methylation have been implicated in several mental disorders, and recently, a number of studies has focused the attention on the possible role of DNA methylation in the mechanisms associated with the development of stress related psychiatric disorders.

However, only few studies have used epigenetic markers as predictors of treatment response, and they are mainly based on targeted approaches examining few epigenetic sites within specific gene loci (for recent literature review see [54]). Among the most studied genes are the Serotonin Transporter (SLC6A4), Brain derived neurotrophic factor (BDNF), Interleukin-11 (IL-11), and Monoamine Oxidase A (MAOA), which have all been shown to interact with environmental risk factors, such as stressful experiences, in increasing the 
vulnerability for depression [56]. In addition to increase disease risk, these genes may also moderate or even mediate therapeutic effects of antidepressant drugs.

Here below we discuss how methylation changes in the candidate genes above reported have been found able to predict antidepressant response.

The SLC6A4 gene has been associated with the vulnerability for depression, and it may also represent a key gene for treatment response, perhaps through epigenetic modifications [59,60,61]. As an example, Kang and colleagues investigated DNA methylation status of several CpG sites within the SLC6A4 gene by examining association between methylation changes in the promoter region with childhood adversities, pre-treatment clinical features and treatment outcomes in 108 MDD patients. However, they did not find any reliable correlation between SLC6A4 methylation and antidepressant response, probably because different kinds of antidepressant drugs with different mechanisms of action were used [62]. Of note, a higher DNA methylation status was found in those subjects with a history of childhood adversities.

A subsequent study conducted by Domschke and collaborators examined the DNA methylation status at nine $\mathrm{CpG}$ sites within the transcriptional control region exon 1A of the SLC6A4 gene in patients suffering from MDD and treated with escitalopram for 6 weeks. They found that the average gene methylation showed a significant association with antidepressant treatment response after 6 weeks: more specifically, DNA hypomethylation determined impaired response to SSRI treatment, whereas higher methylation was associated with a better treatment response [63]. Okada and colleagues examined $81 \mathrm{CpG}$ sites within the SLC6A4 gene and reported that the level of DNA methylation correlated negatively with the severity of illness, and also with treatment response; indeed, the methylation levels of several CpG sites within the promoter region of SLC6A4 gene changed significantly following pharmacological treatment with different antidepressant drugs (paroxetine, fluvoxamine, or milnacipran). Notably, the methylation rate of $\mathrm{CpG} 3$ in patients with better therapeutic responses was significantly higher than in patients with poorer responses [64].

Also the gene encoding for the neurotrophin $B D N F$ has been considered as potential predictor of antidepressant efficacy. Indeed, consistent clinical findings indicated that depressed patients have reduced levels (protein or mRNA) of BDNF in the blood, which are increased after antidepressant treatment; importantly several data reported also a positive correlation between changes in BDNF levels and an amelioration of clinical symptoms, suggesting that BDNF may predict antidepressant efficacy $[15,25,65,66]$.

In line with this, several researchers investigated DNA methylation changes in BDNF gene in order to establish whether they may also predict treatment response. For example, Tadic and collaborators examined the possible correlation between antidepressant response and DNA methylation levels at $12 \mathrm{CpG}$ sites in the promoter region of BDNF exon IV in leukocytes from 39 MDD patients. Interestingly, patients showing a hypomethylation of this region were also those that did not benefit from antidepressant therapy [67]. Gassen and colleagues treated isolated peripheral blood cells from depressed patients with the antidepressant paroxetine and they found that the treatment increased BDNF expression, an effect that was due to reduced activity of DNMT1 and DNA methylation [68]. Moreover, the abundance of BDNF positively correlated with treatment response in patients. 
When considering the gene encoding for the cytokine IL-11, it was shown that its levels were able to predict treatment response in a cohort of MDD patients, and this may be related to epigenetic modifications. Indeed, Powell and colleagues investigated IL-11 DNA methylation in 113 MDD patients randomly selected from the GENDEP sample, treated with escitalopram or nortriptyline for 12 weeks, and found that lower levels of IL11 DNA methylation were associated with better treatment response. However, hypermethylation of $\mathrm{CpG}$ site 5 was specifically associated with the response to escitalopram, whereas hypomethylation of CpG site 4 was specifically associated with the response to nortriptyline [69].

All these studies suggest the potential clinical use of DNA methylation-based biomarkers as predictors of antidepressant response. Moreover, epigenetic modifications could also be key modulators of individual response to antidepressant treatment: in particular, changes in epigenetic modifications during lifespan, provides an attractive model to explain why many pharmacological interventions for depression are not working in some patients $[8,54]$.

\subsection{Histone modifications}

Histones are proteins that package and order the DNA into structural units called nucleosomes. They are the principal protein components of chromatin, acting as spools around which DNA is coiled up, thus playing a role in gene regulation. Histones undergo a number of different post-translational modifications that alter their interaction with DNA and nuclear proteins. The most common chemical modification is acetylation, which is catalyzed by histone acetyl-tranferase (HATs); other modifications include methylation, phosphorylation, ubiquitination, SUMOylation, citrullination and ADP-ribosylation [70].

Several studies, using animal models or human post-mortem samples, have implicated histone modifications in the mechanisms underlying stress responses and the development of depressive phenotypes with the possibility that such changes can be normalized following antidepressant treatment (for a review see [20]) although, only few studies have been conducted in peripheral blood samples.

In one of these studies, peripheral blood histone H3 lysine 27 trimethylation (H3K27me3) levels were measured in 25 treatment-naïve depressed patients before and after treatment with citalopram. Patients who responded to the 8-weeks treatment were found to have a significant decrease in H3K27me3 levels at the promoter IV of the BDNF gene after treatment, an effect that was paralleled by an elevation of BDNF mRNA expression. Interestingly, these changes were not found in the non-responder group, indicating that effective antidepressant treatment modifies the chromatin state of the BDNF gene at peripheral level [71].

In a different study, the authors compared gene expression levels of histone deacetylase 5 (HDAC5) in leukocytes obtained from control subjects and depressed patients who were also assessed before and after 8 weeks of paroxetine treatment. They showed that baseline levels of HDAC5 were significantly higher in drugfree depressed patients, an effect that was normalized by the pharmacological treatment $[20,72]$.

Although not many data are available in the literature, these findings suggest that histone modifications may also represent potential biomarkers useful in the discovery of predictive biomarkers of antidepressant response. 


\section{3 microRNAs (miRNAs)}

Another alternative epigenetic mechanism that appears to be involved in the pathogenesis of psychiatric disorders and in treatment responses is represented by MicroRNAs (miRNAs). MiRNAs are single-stranded, non-coding RNA molecules that belong to a family of 18-25 nucleotides-long small non-coding RNAs. Their biogenesis in mammals is a step process that involves multiple enzyme (for a detailed review [73]) and they have two key roles in the regulation of messenger RNA (mRNA): they act by inducing either degradation or translational silencing of the mRNA they target, but in certain instances, miRNAs may activate translation or even act at the level of transcription by binding to specific gene promoters $[74,75]$.

MiRNAs play an important role in numerous brain mechanisms There is increasing evidence suggesting a key role for miRNAs in neurogenesis [76], neural plasticity and higher brain functioning [77] and they have been associated with neurodegenerative disorders $[78,79]$ as well as psychiatric illnesses [80].

Interestingly recent studies suggested that the action and efficacy of antidepressant drugs treatment may be associated with changes in miRNA expression and function [75]. As an example, Lopez and colleagues measured the blood levels of miR-1202 in healthy controls and also in different independent cohorts of depressed patients, classified into remitters and non-responders, at baseline and after 8 weeks of antidepressant treatment. Responders displayed lower baseline miR-1202 levels as compared with non-responders, and miRNA levels increased following treatment, suggesting that miR-1202 may represent a peripheral biomarker able to predict the treatment response. Noteworthy, this study has also shown a consistent dysregulation of miR-1202 in human postmortem brain tissue, in a well-characterized animal model of depression, in an in vitro model of neural precursor cells and in blood samples from MDD individuals, supporting the role of this miRNA in the pathogenesis of depression [81].

Interestingly, this finding was replicated by the same group in another study that enrolled 2 cohorts of depressed patients treated for 8 weeks with antidepressants (escitalopram or desvenlafaxine or duloxetine). While beyond miR-1202, other miRNAs were found to predict treatment response, the results on miR-16 and miR-135a displayed variability across the cohorts, suggesting that the effects of these two miRNAs may be influenced by other clinical variables. It is worth mentioning that miR-16 and miR-135a have previously been shown to mediate antidepressant actions in humans and animal models of depression $[82,83,84,85]$.

By using a candidate miRNA approach, He and colleagues investigated the expression levels of miRNA-124 in PBMC of 32 depressed patients at baseline and after 8 weeks of treatment with several antidepressants drugs. They observed that the baseline expression levels of miR-124 from PBMCs in depressed patients were significantly higher as compared to controls. In addition, they also found that the expression levels of miR124 were significantly down-regulated after eight weeks of treatment and its levels correlated with treatment response [86].

Enatescu and colleagues performed a pilot study (5 patients) using a whole genome approach to investigate circulating plasma miRNA profiles as potential markers for depression and treatment response. Blood samples were collected at baseline and after 12 weeks of escitalopram treatment and, among the 222 miRNAs detected in blood, the authors identified 40 potentially dysregulated miRNAs, which were modulated by antidepressant 
treatment. Interestingly, these miRNAs were involved in the regulation of pathways such as Wnt signaling, Axon guidance and MAPK signaling that have been suggested to contribute to the mechanism of action of antidepressant drugs [87].

Lopez and colleagues used a sequencing approach to investigated miRNAs as biomarkers of antidepressant response in paired samples from MDD patients enrolled in a large randomized placebo-controlled trial of duloxetine collected before and 8 weeks after treatment. They showed that miR-146a-5p, miR-146b-5p, miR425-3p and miR-24-3p were all down-regulated after antidepressant treatment and that some of their target genes, which were involved in mitogen-activated protein kinase (MAPK) and Wnt signaling pathways were also dysregulated [88].

In summary, while a limited number of studies on miRNAs are currently available (for a review see [89]), they provide preliminary evidence that specific miRNAs may represent useful biomarkers of treatment response, although they must be replicated in larger cohorts. Moreover, these miRNA, and their gene targets, could lead to a better understanding of the mechanisms of antidepressant response. In future perspective miRNAs could also represent a new target of therapeutic intervention.

\section{GENE EXPRESSION AND PROTEIN BLOOD MARKERS AS PREDICTORS OF TREATMENT RESPONSE": FROM CANDIDATE GENE TO HYPOTHESIS FREE APPROACHES"}

\subsection{Transcriptomics and Proteomics}

While for decades, the most accepted hypothesis was based on alterations of monoaminergic systems, this hypothesis fails to explain the latency or lack of response to antidepressant drugs. Emerging theories focus on the dysregulation of inflammatory pathways and neuroplasticity-related processes, and because of this, here we decided to focus on gene expression and protein levels of molecules mainly related to cytokines signalling and neurogenesis/neuroplasticity as possible biomarkers for improving the current strategies of pharmacological intervention.

\subsection{Inflammatory related biomarkers}

A growing body of evidence suggests that pro-inflammatory cytokines like interleukins, chemokines and the Interferon's family molecules have a leading role in the pathophysiology of depression, but also and most importantly, inflammatory markers have been shown to have the potential to predict the outcomes of antidepressants treatments $[25,90,91,92]$.

With this respect, researchers have focused their attention on SNPs for inflammatory genes as well as on the analysis of mRNAs and protein levels in order to identify biomarkers possibly associated with the efficacy of antidepressant drugs (see Table 3). 


\subsubsection{Genetic variants within inflammatory genes}

Recent studies demonstrated that several SNPs within interleukins genes, such as the pro-inflammatory cytokines IL-1beta, IL-6 and IL-11, can influence the antidepressant drugs' response. The IL-1beta polymorphisms have been found to influence the response to antidepressant drugs, where variants within the 2 q14 T-511C locus have been associated, for example, with the response to fluoxetine: in particular, $-511 \mathrm{C} / \mathrm{C}$ homozygotes subjects showed reduced response to fluoxetine as compared to the $-511(\mathrm{C} / \mathrm{T})$ carriers or $(\mathrm{T} / \mathrm{T})$ homozygotes [93]. The same variants have been implicated in the response to other antidepressant drugs: subjects with the $-511 \mathrm{~T} / \mathrm{T}$ genotype showed a faster response to paroxetine as compared to $-511 \mathrm{C} / \mathrm{C}$ subjects, whereas there was no evidence of an association between these variants and the antidepressant mirtazapine [94]. Also SNPs within other loci (rs114643 and rs16944 SNPs) in IL-1beta have been related with antidepressant response, demonstrating that the G/G genotype of both rs114643 and rs16944 SNPs influence the response of a 6-week treatment with different classes of antidepressants [95]. Furthermore, also the rs7801617 in IL-6 gene has been found associated with response to escitalopram [96]; in addition, IL-11 SNP rs1126757 showed a better outcome than G carriers after 12 weeks of escitalopram therapy [96].

Recent studies demonstrated that several SNPs within interleukins genes, such as the pro-inflammatory cytokines IL-1beta, IL-6 and IL-11, can influence the antidepressant drugs' response. The IL-1beta polymorphisms have been found to modulate the response to different antidepressant drugs, such as fluoxetine, paroxetine and mirtazapine $[93,94,95]$. In addition, similar findings were reported also with regard to several SNPs on IL-6 gene and IL-11 [96]. These studies commonly showed how different genetic variants can be correlated with a positive or negative response to the pharmacological therapy. For more detailed information, see Table 3.

\subsection{2 mRNA levels and treatment response}

Several studies have consistently shown an association between higher mRNA levels of inflammatory genes and poor antidepressant response. Our group has shown that high blood mRNA levels of IL-1beta, MIF and TNF-alpha can predict the lack of responsiveness to different drugs, such as escitalopram and nortriptyline [25]. Moreover, a reduction in the levels of the pro-inflammatory cytokines IL-6 and of the GR-associated gene FKBP5 were observed in patients who gain successful benefits from therapy [25]. In line with this, Powell and colleagues, confirmed the presence of high level of TNF-alpha in association with a failure in treatment response, and they also showed that reduced IL-11 mRNA levels were associated with a successful response to escitalopram [97].

Another emerging candidate is the ATP-binding Cassete sub-family F member (ABCF1), a regulator of the inflammatory pathways and of cytokines functions. Powell and colleagues reported increased mRNA levels of this protein in the blood of patients treated with escitalopram and they also showed increased levels of ABCF1 in responder patients after 8 weeks of treatments [98]. 


\subsubsection{Protein levels and treatment response}

A number of meta-analyses reported increased protein levels of several pro-inflammatory cytokines in peripheral blood of depressed patients as compared to controls $[99,100]$ and that changes in cytokines levels during antidepressant treatment correlate with treatment response [101,102,103,104]. For example, increased baseline levels of TNF-alpha correlated with a poor response to amitriptyline [105] and SSRI [106,107] and, in the study of Laquillon and collaborators, baseline level of IL-6 predicted a lack of efficacy of amitriptyline [105]. However, some studies showed inconsistent results regarding the potential role of IL-6 in discriminating between responders and non-responders [108,109]. For instance, higher baseline IL-6 levels were found in patients who responded to SSRI (paroxetine or sertraline) as compared non-responders [110].

Another relevant inflammatory related biomarker for antidepressant response is the $\mathrm{C}$ reactive protein (CRP), whose levels have been found associated with response to different antidepressant drugs by several studies [111,112], suggesting that peripheral blood concentration of CRP might be useful to predict the future therapeutic response. Indeed, it was shown that the SSRI escitalopram is less effective in subjects with high baseline CRP levels, whereas nortriptyline exerted at baseline a higher effect at moderate to high inflammatory conditions [113]. With this respect, it has been assumed that SSRIs may be the most appropriate medication choice for depressed patients with low CRP levels ( $<1 \mathrm{mg} / \mathrm{L})$, whereas subjects with higher levels $(\geq 1 \mathrm{mg} / \mathrm{L})$ may show better clinical outcomes after treatment with dopamine targeting drugs [114]. By contrary, Harley and colleagues showed a positive correlation between a good response to amitriptyline and elevated pretreatment CRP levels [115].

Over the last few years, treatment-resistant patients have also been treated with NMDA receptor antagonist Ketamine. A few studies have been conducted in order to identify possible biomarkers for predicting the response to this new compound. Yang and colleagues has addressed IL-6 as a potential biomarker for Ketamine's antidepressant effect, since baseline levels of this cytokine in responder patients are significantly higher than those in the non-responders group [116]. In addition, Ortiz identified a post-synaptic density protein involved in NMDA receptor functioning, SHANK3, as a potential biomarker, as higher levels of this protein predict an antidepressant response at different time points after Ketamine infusion [117].

In conclusion, the investigation of a panel of inflammatory-related genes, both in term of protein or mRNA levels, holds the potential to represent predictive biomarkers for the clinical response to pharmacological intervention. However, although the role of inflammation in the pathogenesis of depression is well established, the use of inflammatory biomarkers in the clinical setting requires further validation in larger samples.

Table 3. Genetic, mRNA and proteomic biomarker in blood for predicting antidepressant treatment outcomes. 


\section{NEUROPLASTICITY RELATED GENES AND ANTIDEPRESSANT TREATMENT RESPONSE}

Among the molecules with a neurotrophic support and with a potential role in antidepressant efficacy, the neurotrophin Brain Derived Neurotrophic Factor (BDNF) is the one most investigated. BDNF is implicated in the development and maintenance of neural interconnections, in synaptic and dendritic growth, in differentiation of new neurons and synapses and in several brain functions, for example in memory $[118,119,120]$. Several studies pointed out the involvement of BDNF in psychiatric diseases, including depression [121], where it has been widely demonstrated that BDNF mRNA levels are decreased both in the blood and in the brain of depressed patients compared to control subjects [65,122]. Given its multiple roles, BDNF has been suggested as a potential candidate to evaluate the response to antidepressant treatments. Here we will provide a general overview of the main findings on changes on BDNF genetic variants, mRNA levels and also protein levels $[123,124,125]$.

With respect to genetic variants, the most investigated polymorphism is the functional SNP Val66Met (rs6265), which has been associated with alterations in brain morphological features, impaired memories and antidepressant drug response [126,127]. Several studies tried to correlate different genotypes with clinical outcomes and the effects appear to be dependent on the class of antidepressant used. For example, a better clinical improvement after SSRIs treatment was found in Val/Val patients, whereas SNRI/TCAs were more effective in Met carriers [127]. While similar results were found with the SSRI paroxetine [128], opposite findings have been reported by other authors who showed that SSRIs were associated with antidepressant improvement in patients carrying the Met variant after a 6-week treatment [129]. In contrast to the aforementioned studies, other works found inconsistent differences within baseline and after administration of paroxetine (SSRI) and venlafaxine (SNRI) in patients carrying the Val/Val and the Met genotypes [130].

Several studies have also measured BDNF levels in the blood to evaluate whether its levels may be able to predict or mediate the efficacy of antidepressant therapies, although the results are contradictory [131]. Indeed, although several studies showed changes in BDNF levels during antidepressant treatment, with an effect that correlated with clinical response, other studies failed to identify any significant differences in the neurtrophin's levels after antidepressant therapies [108,132]. Most of the studies focused the attention on serum or plasma BDNF levels and only few findings are on mRNA BDNF levels measured in peripheral blood cells [65].

Data on protein levels in the blood are more abundant and they extensively demonstrate the presence of reduced BDNF levels in depressed subjects before treatment, as compared to controls. Importantly, although baseline BDNF levels do not seem to predict the clinical outcome, BDNF changes during treatment are associated with clinical improvement, supporting the notion that drug responsiveness is associated with neuroplastic changes, which are needed to ameliorate clinical symptomatology [65,133]. Polyakova and colleagues in 2014 published a meta-analysis on peripheral BDNF levels in depressed patients treated with antidepressant drug with the median length of treatment was of 6 weeks. The authors, observed reduced BDNF levels in depressed patients at baseline, and showed that circulating BDNF levels were increased in remitters 
and responder patients, but not in non-responder subjects [131]. Another study published consistent findings in elderly patients with pre-treatment depression associated with reduced BDNF levels, which increased upon successful treatment with escitalopram treatment. Interestingly the increase of BDNF levels correlated with symptomatology improvement, supporting the notion that BDNF may indeed be required for antidepressant efficacy [134].

On the other hand, other authors failed to show significant changes of blood BDNF levels in depressed subjects treated with sertraline [132], despite their clinical improvement, suggesting that drug responsiveness may not be unequivocally be associated with an elevation of peripheral BDNF levels $[133,135,136]$.

In summary, based on the available data, it is not so clear whether BDNF could represent a biomarker for depression and treatment response and other factors, such as the Val66Met SNP as well as the presence of epigenetic changes in BDNF, can influence BDNF levels. These variants should be taken into account in larger studies to prove the role of BDNF as biomarker for treatment response. Moreover, a number of technical issues can also influence the results, including the choice of specimen (PBMCs, total blood or leukocytes) for mRNA or protein analysis as well as the time at which blood is collected during or after antidepressant therapy.

\section{METABOLOMICS: A NOVEL GLOBAL APPROACH FOR DRUG RESPONSE PHENOTYPING?}

Metabolomics or metabonomics is the study of the metabolome, the repertoire of biochemical molecules (or small molecules) present in cells, tissues and body fluids [137] that can give information on changes in biochemical and metabolic pathways,and may also providing insights into the complex mechanisms associated with disease and with drug efficacy or side effects $[138,139]$. Metabolites are the final products of interactions between gene expression, protein function and cellular environment. Thus, a specific metabolic phenotype is influenced not only by genotype, but also by environmental factors such as stress, nutrition status, age, gender, disease and co- or pre-administration of other drugs [22]. All these factors contribute to the complexity of the metabolomic analysis.

Metabolites can be assayed through various detection methods, including complex technologies (e.g. nuclear magnetic resonance spectroscopy (NMR), gas chromatography with mass spectroscopy (GC-MS), liquid chromatography with mass spectroscopy LC-MS, and liquid chromatography electrochemical array detection (LCECA) [140].

In the past few years, metabolomic studies have investigated a wide array of metabolites such as lipids, carbohydrates, hormones, aminoacids and even neurotransmitters, with the aim to differentiate depressed from non-depressed patients.

In this regard, a recent review conducted by Hashimoto and colleagues [141] suggested the use of metabolomics to enable the analysis of several small-molecules in different biological fluids, and highlighted the importance of developing specific biomarkers for mood disorders to establish the correct diagnosis and treatment. Studies reviewed in this paper suggest several potential metabolites able to discriminate depressed 
patients from healthy controls, and even also to discriminate sub-symptoms of depression and estimate a degree of suicidal ideation [142]. However further studies are needed to confirm these findings.

At present, metabolomics appear to possess unique and proven advantages for developing biomarkers, and hold great promise for the prediction of patient's response to drug treatment $[18,139,143,144]$.

Here we summarize some of the main findings on metabolome in the context of predictors of antidepressant treatment response.

One of the first metabolomic study in the field of depression and treatment response was carried out by Kaddurah-Daouk and co-workers $[139,144]$ who interrogated baseline metabolite profiles as possible markers associated with a future response to sertraline, or placebo and biomarkers associated to both (placebo and sertraline). The authors used a liquid chromatography electrochemical array (LCEA) platform and found a panel of metabolites specifically in responders as compared to non-responder. As an example, they found that a decrease of 5-methoxytryptamine and an increase in arachidonic acid and $\alpha$-ketoglutarate levels after 1 week of sertraline treatment, were associated with a reduction in symptoms. Four weeks of treatment with sertraline were also associated with decreased levels of lactic acid, BCAAs, valine, leucine and isoleucine.

In another work, they showed that metabolic profiles measured in depressed patients prior to treatment could stratify responders from non-responders to sertraline or placebo [144]. Then they provide detailed mapping of effects of sertraline and placebo on key neurotransmitter pathways after one week and four weeks of treatment; moreover, they correlated biochemical changes with treatment outcomes. Furthermore, they focused on the tryptophan pathway and correlated signalling and they identified early biochemical changes correlated with treatment outcome, providing detailed mapping of the effects produced by sertraline or placebo on key neurotransmitter pathways after one week and four weeks of treatment, correlating biochemical changes with treatment outcomes [145].

By using a pharmacometabolomics approach, Rotroff and collaborators mapped the global metabolic effects of ketamine or its S-enantiomer, esketamine in treatment-refractory MDD patients, and the effects of esketamine on metabolism were tested in the same subjects also following a second exposure administered 4 days later. Both drugs altered metabolites related to tryptophan metabolism (such as indole-3-acetate and methionine) and/or the urea cycle (for example, citrulline, arginine and ornithine) at $2 \mathrm{~h}$ post infusion. In addition, they observed changes in glutamate and circulating phospholipids that significantly correlated with clinical improvement [146].

In another study, among all the metabolites, glycine and glycine dehydrogenase were associated with treatment response to the SSRIs citalopram/escitalopram [147]. These results were confirmed by another study conducted on 529 depressed patients treated with the SSRIs citalopram/escitalopram for 8 weeks. The authors of this study also reported an association between the metabolomic findings with the genetic variations in genes encoding glycine synthesis and degradation enzymes [148].

In a recent study, Gupta and colleagues used a pharmacometabolomics-informed pharmacogenomics research strategy to identify genes associated to metabolites that were also relevant for SSRI response. This "combined" approach made possible to integrate both the role of environmental factors and genomic variability as factors 
influencing the drug responses. They identified 31 metabolites, primarily related to monoamine neurotransmitters, and they also identified SNPs in genes not previously known to be involved in either SSRI response or the regulation of serotonin-related pathways [143].

In light of these data, metabolite profiling holds great promise for the identification of individual metabolites, but most importantly of metabolic pathways involved in antidepressant response. Today only a narrow range of chemical analyses has been performed, but they will be replaced in the future by a more comprehensive metabolic signature. Inclusion of metabolomics data in precision medicine will provide an invaluable layer of data that may allow the integration of the data obtained from other "omics" approach. 


\section{CONCLUSION}

Although different classes of antidepressant drugs acting with different molecular mechanisms are currently available, only the $50 \%$ of patients respond to the first trial, and about the $30 \%$ is not responder to any drugs and become treatment resistance. Thus, there is an urgent need to identify biomarkers that can predict treatment outcome before the patient starts the treatment, in order to identify the best antidepressant for each individual patient and thus to avoid delays in reaching significant symptomatology improvement.

The research of biomarkers in depression is still an ongoing work. Although some potential biomarkers have been identified, they still need to be validated in larger samples. Up to now, most of the studies focused the attention on candidate genes or candidate hypothesis looking at molecules related to inflammation and neuroplasticity, suggesting that cytokines and, more in general, inflammatory related molecules can represent useful predictors of treatment response, whereas neurotrophins like BDNF have been suggested to be more moderators. However, some of these data lead to contrasting results and the potential role of these biomarkers has to be replicated in larger samples before being introduced in the clinical settings. Moreover, the possibility to use high throughput technologies spanning through genome, epigenome, proteome, and metabolome that are now available, can allow the detection of multiple and combined features for predicting antidepressant response. We believe that a combined set of biomarkers including gene or protein expression, cytochrome and metabolites will hold a great potential for the future in predicting treatment response, which will represent a significant advancement for mental health...

\section{EXPERT COMMENTARY}

Major depression is a heterogeneous illness whose aetiology is influenced by a combined contribution of individual genetic variability as well as gene-environment interactions ( $\mathrm{G} \mathrm{X} \mathrm{E)}$.

A crucial challenge for the near future will be to define individual depressed patient on the basis of their symptomatology and severity, as well as the overlap of symptoms across different psychiatric conditions. This could be achieved, at least in part, through the identification of biomarkers associated with 'true' depression, i.e. chronic recurrent depression, and/or its subtypes, including the ones responsive to pharmacological treatment. This represents a critical first step for more effective treatments associated with higher rates of remission, which may ultimately reduce the suffering of patients as well as health care-related costs.

Although different pharmacological therapies are currently available, there is still significant variability in antidepressant treatment response and about the $30 \%$ of patients do not respond to treatments.

This observation highlights the need to identify objectively measurable biomarkers that are able to predict the outcome of antidepressant drugs.

Despite recent findings that we have described in this review, much of the study pertaining to blood-based biomarkers of depression suffers from a number of common weaknesses.

To date, no validated biomarkers have sufficiently proven specific, sensitive and reproducible for clinical application with the possible exception of kits that assess metabolizer status by genotyping cytochrome gene variants. This is due, at least in part, to the heterogeneity among studies (samples analysed - plasma or serum, 
technologies and methods used, inclusion/exclusion criteria of cohort selection, sample size), the shortage of independent replication cohort in which to validate initial findings and the lack of standard operative procedures between researchers (i.e. methods of collecting, storing, processing and analyzing markers). All these elements can indeed impact the ability to draw generalizable conclusions across studies.

Furthermore, up to now, none of the hypothesis-driven studies based on large GWAS or whole-transcriptomic analyses have identified robust biomarkers of antidepressant response, and their findings have been poorly replicated. On the other side, proteomic and metabolomic markers holds great promise for the identification of pathways involved in antidepressant response but they are still limited in number, due to the complex technologies, high costs and technical skills required. Studies focusing on epigenome, have been rarely analyzed, although recent findings suggest they are an interesting path to explore. Indeed, epigenetics may provide new insight into pathophysiology of major depression and may yield novel biomarkers for diagnosis and treatment response. Several different types of epigenetic mechanisms have been implicated to date: they include DNA methylation, post-translational modification of histone proteins and also the regulation of transcription and translation by non-coding RNAs. Some epigenetic modifications, especially DNA methylation, have been considered irreversible in the past. By now it has been shown that even stable chemical modifications, such as DNA methylation, underlie highly dynamic regulation. This potential reversibility makes these mechanisms suitable to be tested as potential biomarkers for treatment response, also because there are increasing evidences that drugs in current therapeutic practice directly or indirectly affect epigenomic status, and this in turn can influence the treatment response.

Some promising results have emerged in the field of inflammation where protein or mRNA levels of proinflammatory cytokines have been reported to predict treatment response, and, if validated by future independent studies in larger cohorts, these may hold promise as targets to be developed within clinical settings. Moreover, advancing technologies might allow to move towards a hypothesis-driven approach to a priori hypothesis that could provide understanding in the pathophysiology of depression and mechanisms of action of antidepressants.

The ultimate goal is the development of a precision medicine in psychiatry that may guide in the identification of a personalized treatment strategy for each patient and in the discovery of new targets for the development of novel antidepressant drugs.

To achieve this goal, it is necessary that future studies take an integrative approach that include clinical assessment, evaluation of environment components as well as a panel of molecular biomarkers. Single traits or feature have a low power and could show low or lack sensibility, sensitivity and reproducibility, however, if several features are taken together they could show a biosignature more sensitive and accurate in predicting response.

\section{FIVE YEARS VIEW}

Despite the fact that several positive findings are described in scientific literature, current biomarker research has some important limitations. Indeed, data are sometime contrasting and inconsistent, possibly due to: 
i) confounding technical issues (i.e. the study design and methodology, quality of starting material);

ii) type of tissue analysed (PBMC, whole blood, serum, plasma, post-mortem brain);

iii) different psychopathological conditions with heterogeneous phenotypes;

iv) treatments and therapies administered (which treatment, dosage, duration of treatment);

v) sample size of the cohorts (too small).

Furthermore, up to now we still need to promote the research focused on the identification of biomarkers that may interact or synergize to determine specific depression phenotypes. Thus, future clinical studies on biomarkers should take an integrative approach in order to identify multiple features (biosignature), which may predict with high accuracy the response to antidepressant therapies.

\section{REFERENCES}

1. American Psychiatric Association. (2013). Diagnostic and statistical manual of mental disorders (5th ed.). Washington, DC: Author.

2. Flint J, Kendler KS. The Genetics of Major Depression. Neuron, 81(5), 1214 (2014).

3. Uher R. The implications of gene-environment interactions in depression: will cause inform cure? Mol Psychiatry, 13(12), 1070-1078 (2008).

4. Keers R, Uher R. Gene-environment interaction in major depression and antidepressant treatment response. Curr Psychiatry Rep, 14(2), 129-137 (2012).

5. Klengel T, Binder EB. Gene $x$ environment interactions in the prediction of response to antidepressant treatment. Int J Neuropsychopharmacol, 16(3), 701-711 (2013).

6. Kovacs D, Gonda X, Petschner P et al. Antidepressant treatment response is modulated by genetic and environmental factors and their interactions. Ann Gen Psychiatry, 13, 17 (2014).

7. Williams LM, Debattista C, Duchemin AM, Schatzberg AF, Nemeroff CB. Childhood trauma predicts antidepressant response in adults with major depression: data from the randomized international study to predict optimized treatment for depression. Transl Psychiatry, 6, e799 (2016).

8. Labermaier C, Masana M, Muller MB. Biomarkers predicting antidepressant treatment response: how can we advance the field? Dis Markers, 35(1), 23-31 (2013).

9. Dunlop BW. Prediction of treatment outcomes in major depressive disorder. Expert Rev Clin Pharmacol, 8(6), 669-672 (2015).

10. Leuchter AF, Cook IA, Marangell LB et al. Comparative effectiveness of biomarkers and clinical indicators for predicting outcomes of SSRI treatment in Major Depressive Disorder: results of the BRITE-MD study. Psychiatry Res, 169(2), 124-131 (2009).

11. Zisook S, Lesser I, Stewart JW et al. Effect of age at onset on the course of major depressive disorder. Am J Psychiatry, 164(10), 1539-1546 (2007).

12. Jeon SW, Kim YK. Inflammation-induced depression: Its pathophysiology and therapeutic implications. J Neuroimmunol, 313, 92-98 (2017).

13. Miller AH, Raison CL. The role of inflammation in depression: from evolutionary imperative to modern treatment target. Nat Rev Immunol, 16(1), 22-34 (2016).

14. Cai S, Huang S, Hao W. New hypothesis and treatment targets of depression: an integrated view of key findings. Neurosci Bull, 31(1), 61-74 (2015). 
15. Cattaneo A, Cattane N, Begni V, Pariante CM, Riva MA. The human BDNF gene: peripheral gene expression and protein levels as biomarkers for psychiatric disorders. Transl Psychiatry, 6(11), e958 (2016).

16. Kennedy SH, Lam RW, McIntyre RS et al. Canadian Network for Mood and Anxiety Treatments (CANMAT) 2016 Clinical Guidelines for the Management of Adults with Major Depressive Disorder: Section 3. Pharmacological Treatments. Can J Psychiatry, 61(9), 540560 (2016).

17. Krystal JH, Sanacora G, Duman RS. Rapid-acting glutamatergic antidepressants: the path to ketamine and beyond. Biol Psychiatry, 73(12), 1133-1141 (2013).

18. Gadad BS, Jha MK, Czysz A et al. Peripheral biomarkers of major depression and antidepressant treatment response: Current knowledge and future outlooks. J Affect Disord, (2017). * The review highlights the biomarkers that are differentially expressed in MDD and treatment response and place a particular emphasis on the most recent progress in advancing technology.

19. Bahn S, Universidade de Cambridge C, Centro Médico Erasmus H et al. Biomarker blood tests for diagnosis and management of mental disorders: focus on schizophrenia. Arch. Clin. Psychiatry (São Paulo), 40(1), 02-09 (2013).

20. Belzeaux R, Lin R, Ju C et al. Transcriptomic and epigenomic biomarkers of antidepressant response. J Affect Disord, (2017).

21. Papakostas GI, Shelton RC, Kinrys G et al. Assessment of a multi-assay, serum-based biological diagnostic test for major depressive disorder: a pilot and replication study. $\mathrm{Mol}$ Psychiatry, 18(3), 332-339 (2013).

22. Bilello JA. Seeking an objective diagnosis of depression. Biomark Med, 10(8), 861-875 (2016).

23. Biomarkers and surrogate endpoints: preferred definitions and conceptual framework. Clin Pharmacol Ther, 69(3), 89-95 (2001).

24. Lam RW, Milev R, Rotzinger S et al. Discovering biomarkers for antidepressant response: protocol from the Canadian biomarker integration network in depression (CAN-BIND) and clinical characteristics of the first patient cohort. BMC Psychiatry, 16, 105 (2016).

25. Cattaneo A, Gennarelli M, Uher R et al. Candidate genes expression profile associated with antidepressants response in the GENDEP study: differentiating between baseline 'predictors' and longitudinal 'targets'. Neuropsychopharmacology, 38(3), 377-385 (2013). ** In this article, the authors identify for the first time three baseline predictors of antidepressant treatment response related to inflammation.

26. Leuchter AF, Cook IA, Hamilton SP et al. Biomarkers to predict antidepressant response. Curr Psychiatry Rep, 12(6), 553-562 (2010).

27. Gururajan A, Clarke G, Dinan TG, Cryan JF. Molecular biomarkers of depression. Neurosci Biobehav Rev, 64, 101-133 (2016).

28. Porcelli S, Fabbri C, Spina E, Serretti A, De Ronchi D. Genetic polymorphisms of cytochrome P450 enzymes and antidepressant metabolism. Expert Opin Drug Metab Toxicol, 7(9), 11011115 (2011). * The authors widely discuss CYP polymorphisms and their effect on antidepressant pharmacokinetic parameters, focusing on clinical practice.

29. Winner JG, Carhart JM, Altar CA, Allen JD, Dechairo BM. A prospective, randomized, double-blind study assessing the clinical impact of integrated pharmacogenomic testing for major depressive disorder. Discov Med, 16(89), 219-227 (2013). 
30. Hall-Flavin DK, Winner JG, Allen JD et al. Utility of integrated pharmacogenomic testing to support the treatment of major depressive disorder in a psychiatric outpatient setting. Pharmacogenet Genomics, 23(10), 535-548 (2013).

31. Perez V, Salavert A, Espadaler J et al. Efficacy of prospective pharmacogenetic testing in the treatment of major depressive disorder: results of a randomized, double-blind clinical trial. BMC Psychiatry, 17(1), 250 (2017).

32. Ingelman-Sundberg M. Genetic polymorphisms of cytochrome P450 2D6 (CYP2D6): clinical consequences, evolutionary aspects and functional diversity. Pharmacogenomics $J, 5(1)$, 613 (2005).

33. Bradford LD. CYP2D6 allele frequency in European Caucasians, Asians, Africans and their descendants. Pharmacogenomics, 3(2), 229-243 (2002).

34. Sawamura K, Suzuki Y, Someya T. Effects of dosage and CYP2D6-mutated allele on plasma concentration of paroxetine. Eur J Clin Pharmacol, 60(8), 553-557 (2004).

35. Yoon YR, Cha IJ, Shon JH et al. Relationship of paroxetine disposition to metoprolol metabolic ratio and CYP2D6*10 genotype of Korean subjects. Clin Pharmacol Ther, 67(5), 567-576 (2000).

36. Ueda M, Hirokane G, Morita S et al. The impact of CYP2D6 genotypes on the plasma concentration of paroxetine in Japanese psychiatric patients. Prog Neuropsychopharmacol Biol Psychiatry, 30(3), 486-491 (2006).

37. Fukuda T, Nishida Y, Zhou Q, Yamamoto I, Kondo S, Azuma J. The impact of the CYP2D6 and CYP2C19 genotypes on venlafaxine pharmacokinetics in a Japanese population. Eur $J$ Clin Pharmacol, 56(2), 175-180 (2000).

38. Ververs FF, Voorbij HA, Zwarts P et al. Effect of cytochrome P450 2D6 genotype on maternal paroxetine plasma concentrations during pregnancy. Clin Pharmacokinet, 48(10), 677-683 (2009).

39. Ng C, Sarris J, Singh A et al. Pharmacogenetic polymorphisms and response to escitalopram and venlafaxine over 8 weeks in major depression. Hum Psychopharmacol, 28(5), 516-522 (2013).

40. Desta Z, Zhao X, Shin JG, Flockhart DA. Clinical significance of the cytochrome P450 2C19 genetic polymorphism. Clin Pharmacokinet, 41(12), 913-958 (2002).

41. Fradette C, Yamaguchi N, Du Souich P. 5-Hydroxytryptamine is biotransformed by CYP2C9, 2C19 and 2B6 to hydroxylamine, which is converted into nitric oxide. Br J Pharmacol, 141(3), 407-414 (2004).

42. Sim SC, Risinger C, Dahl ML et al. A common novel CYP2C19 gene variant causes ultrarapid drug metabolism relevant for the drug response to proton pump inhibitors and antidepressants. Clin Pharmacol Ther, 79(1), 103-113 (2006).

43. De Vos A, van der Weide J, Loovers HM. Association between CYP2C19*17 and metabolism of amitriptyline, citalopram and clomipramine in Dutch hospitalized patients. Pharmacogenomics J, 11(5), 359-367 (2011).

44. Yuce-Artun N, Baskak B, Ozel-Kizil ET et al. Influence of CYP2B6 and CYP2C19 polymorphisms on sertraline metabolism in major depression patients. Int J Clin Pharm, 38(2), 388-394 (2016).

45. Gunes A, Dahl ML. Variation in CYP1A2 activity and its clinical implications: influence of environmental factors and genetic polymorphisms. Pharmacogenomics, 9(5), 625-637 (2008). 
46. Mihara K, Kondo T, Suzuki A et al. Effects of genetic polymorphism of CYP1A2 inducibility on the steady-state plasma concentrations of trazodone and its active metabolite mchlorophenylpiperazine in depressed Japanese patients. Pharmacol Toxicol, 88(5), 267-270 (2001).

47. Gressier F, Rotenberg S, Ait Tayeb AE et al. Tobacco Consumption Concerns With the Use of CYP1A2 Metabolized Antidepressants. Am J Psychiatry, 172(9), 909-910 (2015).

48. Uhr M, Tontsch A, Namendorf C et al. Polymorphisms in the drug transporter gene ABCB1 predict antidepressant treatment response in depression. Neuron, 57(2), 203-209 (2008).

49. Sarginson JE, Lazzeroni LC, Ryan HS, Ershoff BD, Schatzberg AF, Murphy GM, Jr. ABCB1 (MDR1) polymorphisms and antidepressant response in geriatric depression. Pharmacogenet Genomics, 20(8), 467-475 (2010).

50. Schatzberg AF, DeBattista C, Lazzeroni LC, Etkin A, Murphy GM, Jr., Williams LM. ABCB1 Genetic Effects on Antidepressant Outcomes: A Report From the iSPOT-D Trial. Am J Psychiatry, 172(8), 751-759 (2015).

51. Berger SL, Kouzarides T, Shiekhattar R, Shilatifard A. An operational definition of epigenetics. Genes Dev, 23(7), 781-783 (2009).

52. Csoka AB, Szyf M. Epigenetic side-effects of common pharmaceuticals: a potential new field in medicine and pharmacology. Med Hypotheses, 73(5), 770-780 (2009).

53. Boks MP, de Jong NM, Kas MJ et al. Current status and future prospects for epigenetic psychopharmacology. Epigenetics, 7(1), 20-28 (2012).

54. Lisoway AJ, Zai CC, Tiwari AK, Kennedy JL. DNA methylation and clinical response to antidepressant medication in major depressive disorder: A review and recommendations. Neurosci Lett, 669, 14-23 (2018). ** This article reviews the advances made in the relatively new field of pharmaco-epigenetics of antidepressant response, including all published articles examining DNA methylation in association with antidepressant treatment response in Major Depressive Disorder.

55. Vialou V, Feng J, Robison AJ, Nestler EJ. Epigenetic mechanisms of depression and antidepressant action. Annu Rev Pharmacol Toxicol, 53, 59-87 (2013).

56. Menke A, Binder EB. Epigenetic alterations in depression and antidepressant treatment. Dialogues Clin Neurosci, 16(3), 395-404 (2014).

57. Klengel T, Binder EB. Epigenetics of Stress-Related Psychiatric Disorders and Gene $\mathrm{x}$ Environment Interactions. Neuron, 86(6), 1343-1357 (2015).

58. Curradi M, Izzo A, Badaracco G, Landsberger N. Molecular mechanisms of gene silencing mediated by DNA methylation. Mol Cell Biol, 22(9), 3157-3173 (2002).

59. Serretti A, Kato M, De Ronchi D, Kinoshita T. Meta-analysis of serotonin transporter gene promoter polymorphism (5-HTTLPR) association with selective serotonin reuptake inhibitor efficacy in depressed patients. Mol Psychiatry, 12(3), 247-257 (2007).

60. Wankerl M, Miller R, Kirschbaum C, Hennig J, Stalder T, Alexander N. Effects of genetic and early environmental risk factors for depression on serotonin transporter expression and methylation profiles. Transl Psychiatry, 4, e402 (2014).

61. Palma-Gudiel H, Fananas L. An integrative review of methylation at the serotonin transporter gene and its dialogue with environmental risk factors, psychopathology and 5-HTTLPR. Neurosci Biobehav Rev, 72, 190-209 (2017).

62. Kang HJ, Kim JM, Stewart R et al. Association of SLC6A4 methylation with early adversity, characteristics and outcomes in depression. Prog Neuropsychopharmacol Biol Psychiatry, 44, 
23-28 (2013).

63. Domschke K, Tidow N, Schwarte K et al. Serotonin transporter gene hypomethylation predicts impaired antidepressant treatment response. Int $J$ Neuropsychopharmacol, 17(8), 1167-1176 (2014).

64. Okada S, Morinobu S, Fuchikami M et al. The potential of SLC6A4 gene methylation analysis for the diagnosis and treatment of major depression. J Psychiatr Res, 53, 47-53 (2014).

65. Cattaneo A, Bocchio-Chiavetto L, Zanardini R, Milanesi E, Placentino A, Gennarelli M. Reduced peripheral brain-derived neurotrophic factor mRNA levels are normalized by antidepressant treatment. Int J Neuropsychopharmacol, 13(1), 103-108 (2010).

66. Zhou C, Zhong J, Zou B et al. Meta-analyses of comparative efficacy of antidepressant medications on peripheral BDNF concentration in patients with depression. PLoS One, 12(2), e0172270 (2017).

67. Tadic A, Muller-Engling L, Schlicht KF et al. Methylation of the promoter of brain-derived neurotrophic factor exon IV and antidepressant response in major depression. In: $\mathrm{Mol}$ Psychiatry. (England, 2014) 281-283.

68. Gassen NC, Fries GR, Zannas AS et al. Chaperoning epigenetics: FKBP51 decreases the activity of DNMT1 and mediates epigenetic effects of the antidepressant paroxetine. Sci Signal, 8(404), ra119 (2015).

69. Powell TR, Smith RG, Hackinger S et al. DNA methylation in interleukin-11 predicts clinical response to antidepressants in GENDEP. Transl Psychiatry, 3, e300 (2013).

70. Strahl BD, Allis CD. The language of covalent histone modifications. Nature, 403(6765), 4145 (2000).

71. Lopez JP, Mamdani F, Labonte B et al. Epigenetic regulation of BDNF expression according to antidepressant response. In: Mol Psychiatry. (England, 2013) 398-399.

72. Iga J, Ueno S, Yamauchi $\mathrm{K}$ et al. Altered HDAC5 and CREB mRNA expressions in the peripheral leukocytes of major depression. Prog Neuropsychopharmacol Biol Psychiatry, 31(3), 628-632 (2007).

73. $\mathrm{Hu}$ Y, Ehli EA, Boomsma DI. MicroRNAs as biomarkers for psychiatric disorders with a focus on autism spectrum disorder: Current progress in genetic association studies, expression profiling, and translational research. Autism Res, 10(7), 1184-1203 (2017).

74. Younger ST, Corey DR. Transcriptional gene silencing in mammalian cells by miRNA mimics that target gene promoters. Nucleic Acids Res, 39(13), 5682-5691 (2011).

75. Dwivedi Y. Pathogenetic and therapeutic applications of microRNAs in major depressive disorder. Prog Neuropsychopharmacol Biol Psychiatry, 64, 341-348 (2016).

76. Rajman M, Schratt G. MicroRNAs in neural development: from master regulators to finetuners. Development, 144(13), 2310-2322 (2017).

77. Wei CW, Luo T, Zou SS, Wu AS. Research progress on the roles of microRNAs in governing synaptic plasticity, learning and memory. Life Sci, 188, 118-122 (2017).

78. Qiu L, Tan EK, Zeng L. microRNAs and Neurodegenerative Diseases. Adv Exp Med Biol, 888, 85-105 (2015).

79. Viswambharan V, Thanseem I, Vasu MM, Poovathinal SA, Anitha A. miRNAs as biomarkers of neurodegenerative disorders. Biomark Med, 11(2), 151-167 (2017).

80. Luoni A, Riva MA. MicroRNAs and psychiatric disorders: From aetiology to treatment. Pharmacol Ther, 167, 13-27 (2016).

81. Lopez JP, Lim R, Cruceanu C et al. miR-1202 is a primate-specific and brain-enriched 
microRNA involved in major depression and antidepressant treatment. Nat Med, 20(7), 764768 (2014).

82. Baudry A, Mouillet-Richard S, Schneider B, Launay JM, Kellermann O. miR-16 targets the serotonin transporter: a new facet for adaptive responses to antidepressants. Science, 329(5998), 1537-1541 (2010).

83. Launay JM, Mouillet-Richard S, Baudry A, Pietri M, Kellermann O. Raphe-mediated signals control the hippocampal response to SRI antidepressants via miR-16. Transl Psychiatry, 1, e56 (2011).

84. Fiori LM, Lopez JP, Richard-Devantoy S et al. Investigation of miR-1202, miR-135a, and miR-16 in Major Depressive Disorder and Antidepressant Response. Int $J$ Neuropsychopharmacol, 20(8), 619-623 (2017).

85. Issler O, Haramati S, Paul ED et al. MicroRNA 135 is essential for chronic stress resiliency, antidepressant efficacy, and intact serotonergic activity. Neuron, 83(2), 344-360 (2014).

86. He S, Liu X, Jiang K et al. Alterations of microRNA-124 expression in peripheral blood mononuclear cells in pre- and post-treatment patients with major depressive disorder. $J$ Psychiatr Res, 78, 65-71 (2016).

87. Enatescu VR, Papava I, Enatescu I et al. Circulating Plasma Micro RNAs in Patients with Major Depressive Disorder Treated with Antidepressants: A Pilot Study. Psychiatry Investig, 13(5), 549-557 (2016).

88. Lopez JP, Fiori LM, Cruceanu C et al. MicroRNAs 146a/b-5 and 425-3p and 24-3p are markers of antidepressant response and regulate MAPK/Wnt-system genes. Nat Commun, 8, 15497 (2017).

89. Belzeaux R, Lin R, Turecki G. Potential Use of MicroRNA for Monitoring Therapeutic Response to Antidepressants. CNS Drugs, 31(4), 253-262 (2017). ** The Authors give a detailed overview of biomarkers, particularly the major studies that have investigated microRNA in relationship to antidepressant treatment response.

90. Miller AH, Maletic V, Raison CL. Inflammation and its discontents: the role of cytokines in the pathophysiology of major depression. Biol Psychiatry, 65(9), 732-741 (2009).

91. Martino M, Rocchi G, Escelsior A, Fornaro M. Immunomodulation Mechanism of Antidepressants: Interactions between Serotonin/Norepinephrine Balance and Th1/Th2 Balance. Curr Neuropharmacol, 10(2), 97-123 (2012).

92. Hashimoto K. Inflammatory biomarkers as differential predictors of antidepressant response. Int J Mol Sci, 16(4), 7796-7801 (2015).

93. Yu YW, Chen TJ, Hong CJ, Chen HM, Tsai SJ. Association study of the interleukin-1 beta (C-511T) genetic polymorphism with major depressive disorder, associated symptomatology, and antidepressant response. Neuropsychopharmacology, 28(6), 1182-1185 (2003).

94. Tadic A, Rujescu D, Muller MJ et al. Association analysis between variants of the interleukin1 beta and the interleukin-1 receptor antagonist gene and antidepressant treatment response in major depression. Neuropsychiatr Dis Treat, 4(1), 269-276 (2008).

95. Baune BT, Dannlowski U, Domschke K et al. The interleukin 1 beta (IL1B) gene is associated with failure to achieve remission and impaired emotion processing in major depression. Biol Psychiatry, 67(6), 543-549 (2010).

96. Uher R, Perroud N, Ng MY et al. Genome-wide pharmacogenetics of antidepressant response in the GENDEP project. Am J Psychiatry, 167(5), 555-564 (2010).

97. Powell TR, Schalkwyk LC, Heffernan AL et al. Tumor necrosis factor and its targets in the 
inflammatory cytokine pathway are identified as putative transcriptomic biomarkers for escitalopram response. Eur Neuropsychopharmacol, 23(9), 1105-1114 (2013).

98. Powell TR ea. ATP-binding cassette sub-family F member 1 (ABCF1) is identified as a putative therapeutic target of escitalopram in the inflammatory cytokine path... - PubMed NCBI. (2013).

99. Kohler CA, Freitas TH, Maes M et al. Peripheral cytokine and chemokine alterations in depression: a meta-analysis of 82 studies. Acta Psychiatr Scand, 135(5), 373-387 (2017).

100. Liu Y, Ho RC, Mak A. Interleukin (IL)-6, tumour necrosis factor alpha (TNF-alpha) and soluble interleukin-2 receptors (sIL-2R) are elevated in patients with major depressive disorder: a meta-analysis and meta-regression. J Affect Disord, 139(3), 230-239 (2012).

101. Kohler CA, Freitas TH, Stubbs B et al. Peripheral Alterations in Cytokine and Chemokine Levels After Antidepressant Drug Treatment for Major Depressive Disorder: Systematic Review and Meta-Analysis. Mol Neurobiol, (2017).

102. Wiedlocha M, Marcinowicz P, Krupa R et al. Effect of antidepressant treatment on peripheral inflammation markers - A meta-analysis. Prog Neuropsychopharmacol Biol Psychiatry, 80(Pt C), 217-226 (2018).

103. Hannestad J, DellaGioia N, Bloch M. The effect of antidepressant medication treatment on serum levels of inflammatory cytokines: a meta-analysis. Neuropsychopharmacology, 36(12), 2452-2459 (2011).

104. Haapakoski R, Mathieu J, Ebmeier KP, Alenius H, Kivimaki M. Cumulative meta-analysis of interleukins 6 and 1 beta, tumour necrosis factor alpha and C-reactive protein in patients with major depressive disorder. Brain Behav Immun, 49, 206-215 (2015).

105. Lanquillon S, Krieg JC, Bening-Abu-Shach U, Vedder H. Cytokine production and treatment response in major depressive disorder. Neuropsychopharmacology, 22(4), 370-379 (2000).

106. Tuglu C, Kara SH, Caliyurt O, Vardar E, Abay E. Increased serum tumor necrosis factoralpha levels and treatment response in major depressive disorder. Psychopharmacology (Berl), 170(4), 429-433 (2003).

107. Eller T, Vasar V, Shlik J, Maron E. Pro-inflammatory cytokines and treatment response to escitalopram in major depressive disorder. Prog Neuropsychopharmacol Biol Psychiatry, 32(2), 445-450 (2008).

108. Ninan PT, Shelton RC, Bao W, Guico-Pabia CJ. BDNF, interleukin-6, and salivary cortisol levels in depressed patients treated with desvenlafaxine. Prog Neuropsychopharmacol Biol Psychiatry, 48, 86-91 (2014).

109. Manoharan A, Rajkumar RP, Shewade DG, Sundaram R, Muthuramalingam A, Paul A. Evaluation of interleukin-6 and serotonin as biomarkers to predict response to fluoxetine. Hum Psychopharmacol, 31(3), 178-184 (2016).

110. Yoshimura R, Hori H, Ikenouchi-Sugita A et al. Plasma levels of interleukin-6 and selective serotonin reuptake inhibitor response in patients with major depressive disorder. Hum Psychopharmacol, 28(5), 466-470 (2013).

111. Howren MB ea. Associations of depression with C-reactive protein, IL-1, and IL-6: a metaanalysis. - PubMed - NCBI. (2009).

112. Raison $\mathrm{CL}$ ea. A randomized controlled trial of the tumor necrosis factor antagonist infliximab for treatment-resistant depression: the role of baseline inflammat... - PubMed NCBI. (2013).

113. Uher R, Tansey KE, Dew T et al. An inflammatory biomarker as a differential predictor of 
outcome of depression treatment with escitalopram and nortriptyline. Am J Psychiatry, 171(12), 1278-1286 (2014).

114. Miller AH, Trivedi MH, Jha MK. Is C-reactive protein ready for prime time in the selection of antidepressant medications? Psychoneuroendocrinology, 84, 206 (2017).

115. Harley J, Luty S, Carter J, Mulder R, Joyce P. Elevated C-reactive protein in depression: a predictor of good long-term outcome with antidepressants and poor outcome with psychotherapy. In: J Psychopharmacol. (United States, 2010) 625-626.

116. Yang JJ, Wang N, Yang C, Shi JY, Yu HY, Hashimoto K. Serum interleukin-6 is a predictive biomarker for ketamine's antidepressant effect in treatment-resistant patients with major depression. Biol Psychiatry, 77(3), e19-e20 (2015).

117. Ortiz R, Niciu MJ, Lukkahati N et al. Shank3 as a potential biomarker of antidepressant response to ketamine and its neural correlates in bipolar depression. J Affect Disord, 172, 307 311 (2015).

118. Begni V, Riva MA, Cattaneo A. Cellular and molecular mechanisms of the brain-derived neurotrophic factor in physiological and pathological conditions. Clin Sci (Lond), 131(2), 123-138 (2017).

119. Sasi M, Vignoli B, Canossa M, Blum R. Neurobiology of local and intercellular BDNF signaling. Pflugers Arch, 469(5-6), 593-610 (2017).

120. Park H, Poo MM. Neurotrophin regulation of neural circuit development and function. Nat Rev Neurosci, 14(1), 7-23 (2013).

121. Hempstead BL. Brain-Derived Neurotrophic Factor: Three Ligands, Many Actions. Trans Am Clin Climatol Assoc, 126, 9-19 (2015).

122. Hong W, Fan J, Yuan C et al. Significantly decreased mRNA levels of BDNF and MEK1 genes in treatment-resistant depression. Neuroreport, 25(10), 753-755 (2014).

123. Amare AT, Schubert KO, Baune BT. Pharmacogenomics in the treatment of mood disorders: Strategies and Opportunities for personalized psychiatry. Epma j, 8(3), 211-227 (2017).

124. Fabbri C, Di Girolamo G, Serretti A. Pharmacogenetics of antidepressant drugs: an update after almost 20 years of research. Am J Med Genet B Neuropsychiatr Genet, 162b(6), 487520 (2013).

125. Niitsu T, Fabbri C, Bentini F, Serretti A. Pharmacogenetics in major depression: a comprehensive meta-analysis. Prog Neuropsychopharmacol Biol Psychiatry, 45, 183-194 (2013).

126. Chen ZY, Bath K, McEwen B, Hempstead B, Lee F. Impact of genetic variant BDNF (Val66Met) on brain structure and function. Novartis Found Symp, 289, 180-188; discussion 188-195 (2008).

127. Colle R, Gressier F, Verstuyft C et al. Brain-derived neurotrophic factor Val66Met polymorphism and 6-month antidepressant remission in depressed Caucasian patients. $J$ Affect Disord, 175, 233-240 (2015).

128. Murphy GM, Jr., Sarginson JE, Ryan HS, O'Hara R, Schatzberg AF, Lazzeroni LC. BDNF and CREB1 genetic variants interact to affect antidepressant treatment outcomes in geriatric depression. Pharmacogenet Genomics, 23(6), 301-313 (2013).

129. Yan T, Wang L, Kuang W et al. Brain-derived neurotrophic factor Val66Met polymorphism association with antidepressant efficacy: a systematic review and meta-analysis. Asia Pac Psychiatry, 6(3), 241-251 (2014).

130. Shen X, Qian M, Yuan Y et al. [Research on association of BDNF gene Val66Met 
polymorphism with efficacy of antidepressants and plasma BDNF level]. Zhonghua Yi Xue Yi Chuan Xue Za Zhi, 31(2), 196-200 (2014).

131. Polyakova M, Stuke K, Schuemberg K, Mueller K, Schoenknecht P, Schroeter ML. BDNF as a biomarker for successful treatment of mood disorders: a systematic \& quantitative metaanalysis. $J$ Affect Disord, 174, 432-440 (2015). * This article reviews the role of BDNF as biomarker for antidepressant treatment and critically discuss the its clinical meaning, focusing on the need of defining optimal time points for measurement.

132. Brunoni AR, Machado-Vieira R, Zarate CA, Jr. et al. BDNF plasma levels after antidepressant treatment with sertraline and transcranial direct current stimulation: results from a factorial, randomized, sham-controlled trial. Eur Neuropsychopharmacol, 24(7), 1144-1151 (2014).

133. Brunoni AR, Lopes M, Fregni F. A systematic review and meta-analysis of clinical studies on major depression and BDNF levels: implications for the role of neuroplasticity in depression. Int J Neuropsychopharmacol, 11(8), 1169-1180 (2008).

134. Martocchia A, Curto M, Scaccianoce S et al. Effects of escitalopram on serum BDNF levels in elderly patients with depression: a preliminary report. Aging Clin Exp Res, 26(4), 461-464 (2014).

135. Molendijk ML ea. Serum BDNF concentrations as peripheral manifestations of depression: evidence from a systematic review and meta-analyses on 179 associations ( $N=94 \ldots$ - PubMed - NCBI. (2014).

136. Sen $\mathrm{S}$ ea. Serum brain-derived neurotrophic factor, depression, and antidepressant medications: meta-analyses and implications. - PubMed - NCBI. (2008).

137. Beger RD, Dunn W, Schmidt MA et al. Metabolomics enables precision medicine: "A White Paper, Community Perspective". Metabolomics, 12(10), 149 (2016).

138. Kaddurah-Daouk R, Kristal BS, Weinshilboum RM. Metabolomics: a global biochemical approach to drug response and disease. Annu Rev Pharmacol Toxicol, 48, 653-683 (2008).

139. Kaddurah-Daouk R, Bogdanov MB, Wikoff WR et al. Pharmacometabolomic mapping of early biochemical changes induced by sertraline and placebo. Transl Psychiatry, 3, e223 (2013).

140. Quinones MP, Kaddurah-Daouk R. Metabolomics tools for identifying biomarkers for neuropsychiatric diseases. Neurobiol Dis, 35(2), 165-176 (2009).

141. Hashimoto K. Metabolomics of Major Depressive Disorder and Bipolar Disorder: Overview and Future Perspective. Adv Clin Chem, 84, 81-99 (2018).

142. Setoyama D, Kato TA, lisoway R et al. Plasma Metabolites Predict Severity of Depression and Suicidal Ideation in Psychiatric Patients-A Multicenter Pilot Analysis. PLoS One, 11(12), e0165267 (2016)

143. Gupta M, Neavin D, Liu D et al. TSPAN5, ERICH3 and selective serotonin reuptake inhibitors in major depressive disorder: pharmacometabolomics-informed pharmacogenomics. Mol Psychiatry, 21(12), 1717-1725 (2016).

144. Kaddurah-Daouk R, Boyle SH, Matson W et al. Pretreatment metabotype as a predictor of response to sertraline or placebo in depressed outpatients: a proof of concept. Transl Psychiatry, 1 (2011).

145. Zhu H, Bogdanov MB, Boyle SH et al. Pharmacometabolomics of response to sertraline and to placebo in major depressive disorder - possible role for methoxyindole pathway. PLoS One, $8(7)$, e68283 (2013).

146. Rotroff DM, Corum DG, Motsinger-Reif A et al. Metabolomic signatures of drug response 
phenotypes for ketamine and esketamine in subjects with refractory major depressive disorder: new mechanistic insights for rapid acting antidepressants. Transl Psychiatry, 6(9), e894 (2016).

147. Ji Y, Hebbring S, Zhu $\mathrm{H}$ et al. Glycine and a glycine dehydrogenase (GLDC) SNP as citalopram/escitalopram response biomarkers in depression: pharmacometabolomicsinformed pharmacogenomics. Clin Pharmacol Ther, 89(1), 97-104 (2011).

148. Abo R, Hebbring S, Ji Y et al. Merging pharmacometabolomics with pharmacogenomics using '1000 Genomes' single-nucleotide polymorphism imputation: selective serotonin reuptake inhibitor response pharmacogenomics. Pharmacogenet Genomics, 22(4), 247-253 (2012). 